\title{
The use of antioxidants against high ferritin-induced oxidation in COVID-19 patients: a biochemical approach
}

\author{
Mustafa Şahin ${ }^{1}$ (D)
}

Received: 24 April 2020 / Accepted: 27 August 2020 / Published online: 11 September 2020

(c) Società Italiana di Medicina Interna (SIMI) 2020

\section{Dear Editor,}

The novel Coronavirus (SARS-CoV-2) outbreak, which began in Wuhan, China, in December 2019, was declared a pandemic by the World Health Organization (WHO) and the disease was named COVID-19. As of August 20, 2020, the disease was reported in 213 countries, with 22,613,985 cases and 791,709 deaths around the world. The outbreak is still spreading; the number of cases, the death rate, and the number of patients in intensive care units are increasing. However, no therapeutic agents or vaccines effective against coronavirus have been announced. Research about different treatment options is ongoing around the world to end the outbreak.

Some laboratory tests, such as CK, LDH, D-dimer, ESR, CRP, procalcitonin and ferritin, have been shown to detect abnormalities in COVID-19 patients. Ferritin is known as an acute phase reactant, but evaluation of it as an acute phase reactant alone may be a deficiency for prognosis in this group of patients. In recent years, increasing evidence supports the idea that circulating high ferritin not only reflects the acute phase response, but also plays a critical role in inflammation. Ferritin is an important intracellular iron storage protein and has two subunits, $\mathrm{H}$ and $\mathrm{L}$. $\mathrm{H}$-ferritin plays a role not only in immunomodulatory functions but also in pro-inflammatory activity that results in the induction of expression of different inflammatory mediators, including IL-1 $\beta$ [1].

The concentration of free ferritin in circulation increases as a result of the binding of coronavirus to the iron molecule that binds oxygen in the hemoglobin molecule. Thus, the iron ion becomes a free molecule and the hemoglobin

Mustafa Şahin

mustafa.sahin57@saglik.gov.tr

1 Department of Medical Biochemistry, Hitit University Erol Olçok Training and Research Hospital, Çorum, Turkey 19300 molecule cannot bind to oxygen. Thus, oxygen saturation is reduced. There is no data, yet that the virus interacts with the iron ion. Otherwise, the formation of free oxygen radicals increases with the increased concentration of ferritin, and typical oxidant effects occur in tissues and organs. Reactive oxygen species (ROS) play a key role in the acute respiratory distress syndrome (ARDS) pathophysiology in COVID19 and constitute the basis of the injury process. Increased free radical formation caused by high ferritin levels can be a marker of poor prognosis in these patients.

However, considering the cytokine storm in COVID-19 patients, hyperferritinemia can be considered to be only part of inflammation and ROS formation, or one of many factors contributing to inflammation and ROS formation. It is not accurate to show that only hyperferritinemia is responsible for all inflammation in COVID-19 patients. Liu et al. emphasized that iron ions are necessary in replication and transcription of coronavirus in the host cell, and noted that iron ions are an important factor for poor prognosis in virus infections. Therefore, they suggested that iron chelation may be an alternative useful adjuvant in the treatment of COVID-19, based on experience from other viral infections [2]. Strategies about iron metabolism may play key roles for the success of COVID-19 therapy. Researchers demonstrated that non-structural proteins of the coronavirus, orf1ab, ORF10 and ORF3a, coordinated to attack heme on the 1-beta chain of hemoglobin, dissociating iron to form porphyrin. Deoxyhemoglobin is more vulnerable to virus attacks than oxidized hemoglobin. This mechanism of the virus inhibits the normal metabolic pathway of heme and induces symptoms of the disease [1].

In computed tomography examinations of COVID-19 patients, ground-glass opacity prevails along with the contribution of free oxygen radicals to this pathology (Fig. 1). Free radical damage in the figure is schematized in COVID19 pathology. There are lots of studies in the literature showing and explaining the effect of antioxidants such as vitamin $\mathrm{C}$ and vitamin $\mathrm{E}$ on the treatment of pneumonia. 


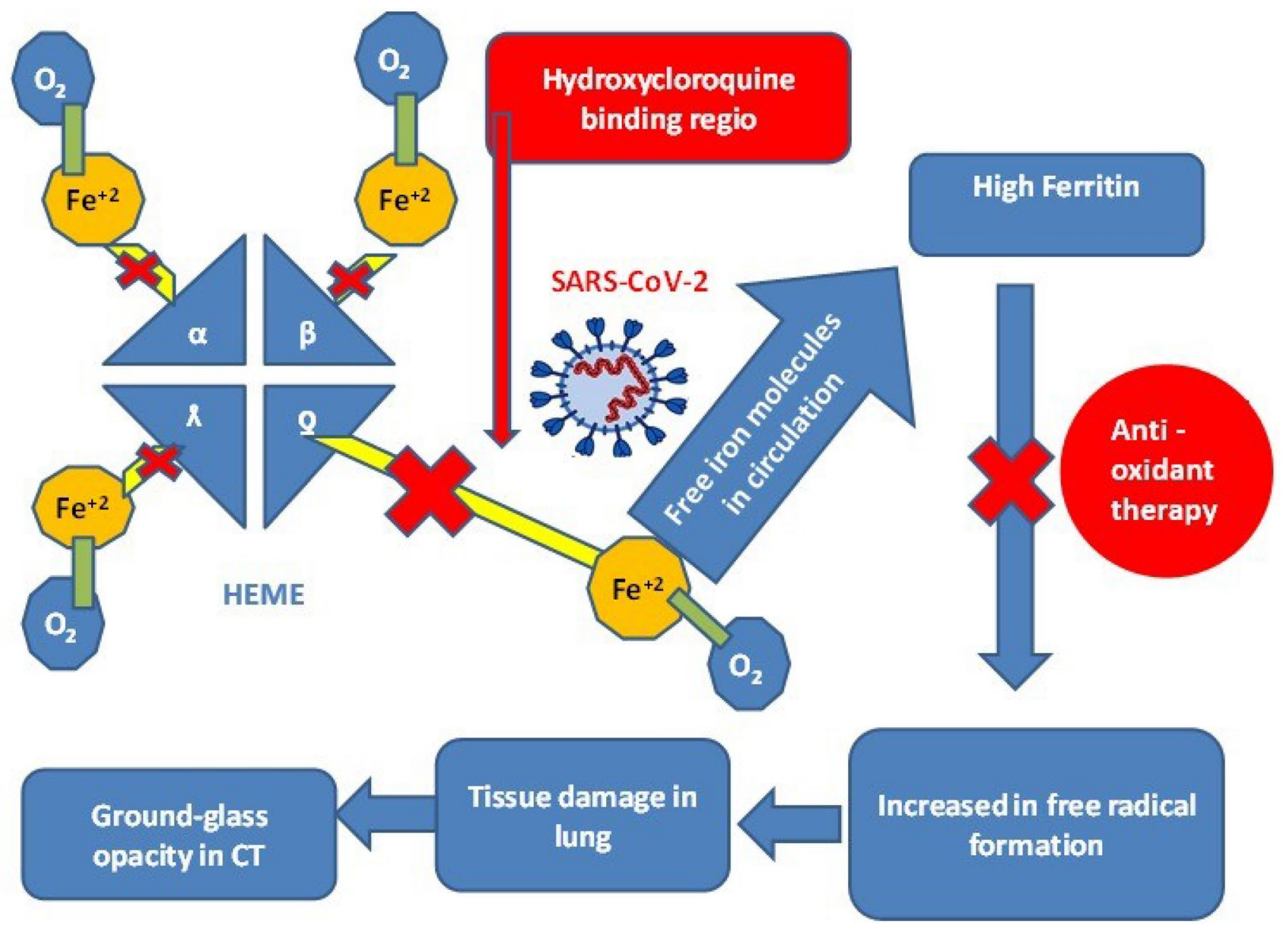

Fig. 1 Relationship between SARS-CoV-2, high iron molecules and antioxidant therapy. SARS-CoV-2 binds to the region of the heme molecule where the iron ion must bind; thus, the iron ion cannot bind to the heme molecule and remains free. Hydroxychloroquine could provide protection by shutting down the region where the virus binds. Anti-oxidant therapy can reduce the formation of free radicals induced by high ferritin

expression, improves alveolar fluid clearance, and acts as an antioxidant when used as a parenteral agent in high doses. The therapeutic role of anti-oxidants in sepsis/ intensive care unit patients was shown in many studies. But there is still debate over combination treatments [4]. The use of vitamin C, a water-soluble, easily excreted molecule with no adverse effect, in COVID-19 patients can offer significant therapeutic opportunities.

Currently, it is stated that the hydroxychloroquine molecule binds to the region where coronavirus binds to the hemoglobin molecule, but there is no clear treatment success. In addition to the current treatment of COVID-19 patients, supplements related to free radical-antioxidant balance can be administered. Preparations containing high doses of vitamin $\mathrm{C}$ can turn off antioxidant deficiency in the body and contribute to repair of damage caused by increased free oxygen radicals. All other antioxidant molecules, but mainly vitamin $\mathrm{C}$, can be added to the treatment protocol of COVID-19 patients. Antioxidant 
vitamins are used to inhibit the hazardous effects of radicals.

Hemila et al. reported that high-dose intravenous vitamin $\mathrm{C}$ infusions (e.g., $200 \mathrm{mg} / \mathrm{kg}$ body weight/day,) shortened the intensive care unit stay by $7.8 \%$, accompanied by a significant reduction in the mortality rate [3]. Additionally, oral vitamin $\mathrm{C}$ (e.g., $6 \mathrm{~g}$ daily) was able to reduce viral infection risk or improve symptoms. High-dose intravenous vitamin $\mathrm{C}$ has also been successfully used in the treatment of 50 moderate to severe COVID-19 patients in China. The doses used varied between 10 and $20 \mathrm{~g}$ per day, given over a period of $8-10 \mathrm{~h}$. The oxygenation index improved in real time and all the patients were eventually cured and discharged [5].

In COVID-19 patients, clinical trials of antioxidant treatments may be supported, especially since vitamin $\mathrm{C}$ is a water-soluble, nontoxic vitamin with a wide confidence interval. In addition, vitamin $\mathrm{C}$ can be used for protective or prophylactic purposes during the COVID-19 pandemic. We think that the free radical-reactive oxygen radicals increase caused by ferritin, which increases due to the free iron ions, can be blocked by the addition of antioxidant treatment for COVID-19 patients. Vitamin C may have the potential to be added to the support treatment given to COVID-19 patients. This paper is to encourage academic discussion and the correctness of the theory needs confirmation via conduction of appropriate invitro and in vivo studies.

\section{Compliance with ethical standard}

Conflict of interest The author declares no conflict of interest.
Human and animal rights This article does not contain any studies with human participants or animals performed by any of the authors.

Informed consent For this type of article, no formal consent is required.

\section{References}

1. Ruddell RG, Le Hoang D, Barwood JM, Rutherford PS, Piva TJ, Watters DJ et al (2009) Ferritin functions as a proinflammatory cytokine via iron-independent protein kinase $\mathrm{C}$ zeta/ nuclear factor kappa B-regulated signaling in rat hepatic stellate cells. Hepatology 49:887-900

2. Liu W, Zhang S, Nekhai S, Liu S (2020) Depriving 1ron supply to the virus represents a promising adjuvant therapeutic against viral survival. Curr Clin Microbiol Rep. https://doi.org/10.1007/ s40588-020-00140-w

3. Hemilä H (1997) Vitamin C intake and susceptibility to pneumonia. Pediatr Infect Dis J 16:836-837

4. Fowler AA, Syed AA, Knowlson S, Sculthorpe R, Farthing D, DeWilde C, Farthing CA, Larus TL, Martin E, Brophy DF, Gupta S, Fisher BJ, Natarajan R (2014) Phase I safety trial of intravenous ascorbic acid in patients with severe sepsis. J Transl Med 12: 32 https://doi.org/10.1186/1479-5876-12-32

5. Shanghai Expert Panel, cited on Mar 23. https://mp.weixin.qq. $\mathrm{com} / \mathrm{s}$ ?__biz= MzA3Nzk5Mzc5MQ==\& $\mathrm{mid}=2653620168 \&$ $\mathrm{idx}=1 \& \mathrm{sn}=2352823 \mathrm{~b} 79 \mathrm{a} 3 \mathrm{cc} 42 \mathrm{e} 4822$ 9a0c38f65e0\&chksm $=$ 84962598 b3e 1 ac8effb763e3ddb4858435dc7aa947a8f41790e 8df2bca34c20e6ffea64cd191\#rd; 2020

Publisher's Note Springer Nature remains neutral with regard to jurisdictional claims in published maps and institutional affiliations. 